\title{
The PIN auxin efflux facilitator network controls growth and patterning in Arabidopsis roots
}

\author{
Ikram Blilou ${ }^{1}$, Jian Xu ${ }^{1 \star}$, Marjolein Wildwater ${ }^{1 \star}$, Viola Willemsen ${ }^{1 \star}$, Ivan Paponov ${ }^{2}$, Jiři Friml $^{3}$, Renze Heidstra ${ }^{1}$, Mitsuhiro Aida ${ }^{1}$, \\ Klaus Palme $^{2}$ \& Ben Scheres ${ }^{1}$ \\ ${ }^{1}$ Department of Molecular Genetics, Utrecht University, Padualaan 8, 3584CH Utrecht, The Netherlands \\ ${ }^{2}$ Institut für Biologie II , Universität Freiburg, Schänzlestrasse1, 79104, Freiburg, Germany \\ ${ }^{3}$ Zentrum für Molekularbiologie der Pflanzen, Universität Tübingen, Auf der Morgenstelle 3, 72076 Tübingen, Germany \\ ${ }^{*}$ These authors contributed equally to this work
}

Local accumulation of the plant growth regulator auxin mediates pattern formation in Arabidopsis roots and influences outgrowth and development of lateral root- and shoot-derived primordia. However, it has remained unclear how auxin can simultaneously regulate patterning and organ outgrowth and how its distribution is stabilized in a primordium-specific manner. Here we show that five PIN genes collectively control auxin distribution to regulate cell division and cell expansion in the primary root. Furthermore, the joint action of these genes has an important role in pattern formation by focusing the auxin maximum and restricting the expression domain of PLETHORA (PLT) genes, major determinants for root stem cell specification. In turn, PLT genes are required for PIN gene transcription to stabilize the auxin maximum at the distal root tip. Our data reveal an interaction network of auxin transport facilitators and root fate determinants that control patterning and growth of the root primordium.

In Arabidopsis root development, a distal auxin maximum correlates with pattern formation and the orientation and extent of cell division ${ }^{1}$. Inhibition of polar auxin transport strongly affects these processes $^{1}$. The initiation of lateral roots and leaf primordia is also associated with changes in auxin transport $\mathrm{t}^{2-4}$. These observations point to polar auxin transport as a major factor in organ formation. Recent analyses of the Arabidopsis PIN proteins revealed their association with auxin maxima in distal domains of organ primor$\mathrm{dia}^{2-4}$. The PIN genes are thought to encode components of the auxin efflux machinery mediating polar auxin transport, as concluded from the polar localization of PIN membrane proteins and auxin uptake experiments ${ }^{5-11}$. PIN proteins might participate directly in auxin transport or help in the assembly of other proteins with efflux activity such as the AtMDR/PGP proteins ${ }^{12,13}$. Mutations in the ARF-GEF GNOM, required for vesicle transport of PIN1, also lead to developmental defects that resemble those caused by interfering with auxin transport ${ }^{14-16}$.

All single pin mutants described so far display different weak phenotypes in primary roots ${ }^{4-8}$, in contrast with gnom mutants and polar auxin transport inhibitor treatments. Here, we investigate the collective contribution of five PIN genes in the control of cell division and cell expansion during root outgrowth. Furthermore, we assess how the PLETHORA (PLT) genes, which encode auxin-inducible AP2-domain putative transcription factors necessary and sufficient for stem cell specification in the root primordium $^{17}$, respond to and regulate PIN gene activity during pattern formation.

\section{PIN protein localization is changed in pin mutants}

The PIN proteins described so far are expressed in specific but overlapping regions of the root meristem ${ }^{6-8}$. PIN1 mainly resides at the basal end of the vascular cells ${ }^{8}$ but weak PIN1 signals can be detected in the epidermis and the cortex (Fig. 1b). PIN2 localizes apically in epidermal and lateral root cap cells and predominantly basally in cortical cells ${ }^{6}$ (Fig. 1e, f). PIN3 is expressed without pronounced polarity in tiers two and three of the columella cells, at the basal side of vascular cells and to the lateral side of pericycle cells of the elongation zone $\mathrm{e}^{7}$ (Fig. 1h, i). PIN4 is detected around the quiescent centre and cells surrounding it, and localizes basally in provascular cells ${ }^{8}$ (Fig. 1k). PIN7 resides at lateral and basal membranes of provascular cells in the meristem and elongation zone, whereas in the columella cells it coincides with the PIN3 domain (Fig. 1j).

Auxin distribution appears to be altered in pin mutants ${ }^{1,8}$ and differences in auxin homeostasis affect PIN2 expression ${ }^{18}$. We therefore determined whether pin mutants have altered expression of remaining PIN proteins. In pin3pin4pin7, enhanced PIN1 protein was detected in lateral-basal membranes of the endodermis (Fig. 1c, d). Moreover, ectopic PIN2 protein was detected at the basal end of provascular cells that normally express PIN3 and PIN7 (Fig. 1g). PIN4 expression expands to tier three of the columella cells in pin3 single mutants and pin2pin3 double mutants with a membrane localization similar to PIN3 in the wild type (Fig. 11 and data not shown). In pin3pin7, PIN4 expands to the lateral root cap (Fig. 1m).

Our data show that defects in pin mutants can be masked by ectopic activity of the remaining PIN genes. A comprehensive mutant analysis is therefore necessary to uncover full gene function. To this end, we generated all mutant combinations for PIN1, PIN2, PIN3, PIN4 and PIN7, which group together within the eightmember PIN gene family. We verified phenotypes in independent allelic combinations to exclude influences of background modifiers (Supplementary Table 1).

PIN genes control cell division zone size in the root meristem Classical experiments revealed that externally added auxins can stimulate cell division ${ }^{19}$ but it is unknown whether internal auxin distribution regulates cell division in primordia. PIN genes are required for outgrowth of all organs $s^{2,3,20}$, and we assessed whether this reflected their contribution to the in vivo regulation of cell division. In roots, oriented cell divisions accompanied by a low rate of cell expansion occur in the distal meristem zone (Fig. 2A).

pin1 and pin 2 single mutants display a slight reduction of root 
length and root meristem size (Supplementary Fig. 2) whereas pin3, pin 4 and pin 7 single mutants only display subtle division defects in the quiescent centre and columella root cap $^{8}$ (Supplementary Fig. 1d-h). Most double-mutant combinations show additive defects in orientation of cell division, root length and root meristem size (Supplementary Figs 1 and 2). However, pin1pin2 and all triple and quadruple mutants containing pin2 show more-than-additive reduction in root size and root meristem size (Fig. 2B, C and Supplementary Fig. 2a, b), suggesting that PIN2 plays a pivotal role in cell division control. The pin1pin2 double mutant suggests that the role of PIN2 in meristem size control is masked in the pin2 single mutant mainly by the activity of PIN1 in the PIN2 domain (Fig. 1b). PIN2 is a main component for mediating proximal (basipetal) auxin transport (Fig. 1e, f, n), which implies that basipetal transport to meristematic cells has a critical role in meristem length regulation. Consistent with the notion of auxin shortage in the meristem

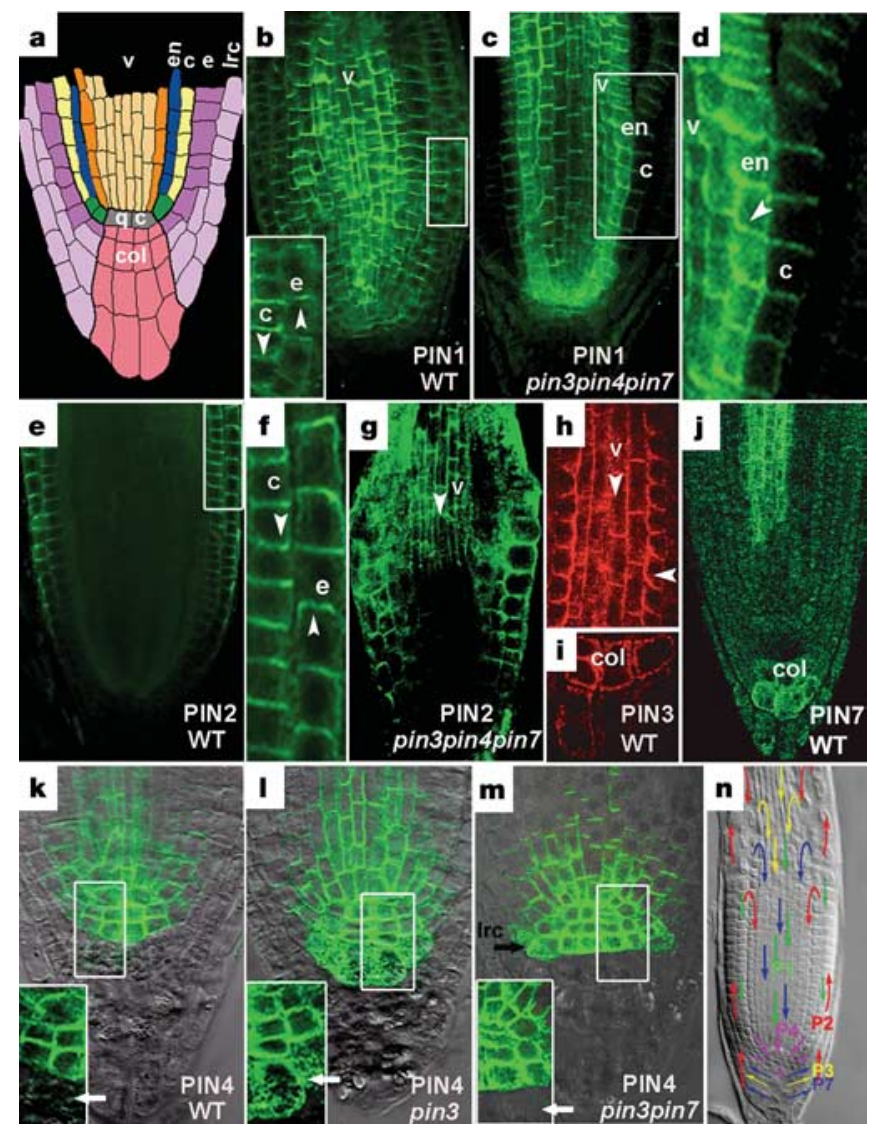

Figure 1 PIN expression and protein localization in roots of wild-type (WT) and pin mutant Arabidopsis. a, Arabidopsis root meristem with columella (col), quiescent centre (qc), lateral root cap (Irc), epidermis (e), cortex (c), endodermis (en) and vascular bundle (v). b-d, PIN1 immunolocalization: b, wild type; inset shows boxed area enlarged. Arrowheads depict polar localization. c, d, pin3pin4pin7 (Allelic Combination 1(AC1)); boxed area in $\mathbf{c}$ is enlarged in $\mathbf{d}$. Arrowheads in $\mathbf{d}$, PIN1 upregulation in the endodermis. $\mathbf{e - g}$, PIN2 protein: e, f, Wild type; boxed area in $\mathbf{e}$ is enlarged in $\mathbf{f}$. Arrowheads in $\mathbf{f}$, apical PIN2 in the epidermis and basal in the cortex. $\mathbf{g}$, pin3pin4pin7 ${ }^{\text {(Ac1); }}$ arrowhead shows ectopic expression. h, i, PIN3 protein in wild-type root meristem. Arrowheads in h, PIN3 in the pericycle. j, PIN7-green fluorescent protein (GFP) fusion. $\mathbf{k}-\mathbf{m}$, PIN4 protein: $\mathbf{k}$, wild type; white arrow, wild-type differentiated columella cell without PIN4. I, pin3 (white arrow); $\mathbf{m}$, pin3pin $7^{\text {(AC1): }}$ black arrow, PIN4 expansion to the lateral root cap; white arrow as in k. Insets: enlargements of boxed areas showing details of PIN4 localization.

$\mathbf{n}$, Localization of PIN proteins suggests auxin transport routes. PIN1 (green), PIN2 (red), PIN3 (yellow), PIN4 (violet) and PIN7 (blue). Immunolocalization signals are green in $\mathbf{b}-\mathbf{g}$ and $\mathbf{k}-\mathbf{m}$ and red in $\mathbf{h}$, i. zone, treatment with auxins restored meristem size of pin1pin2 and pin2pin3pin7 to that of wild type (data not shown). Our data substantiate physiological evidence indicating a role for basipetal auxin transport in root growth ${ }^{21,22}$ and identify control of cell division as a major factor in this process.

The extreme reduction in root meristem size in pin 2 mutant combinations is reminiscent of the phenotype obtained upon diphtheria toxin-mediated genetic ablation of root cap cells ${ }^{23}$. In such plants we found high DR5::GUS accumulation in the provascular tissue (Fig. 2E, F), supporting the notion that lateral auxin redistribution does not occur in the absence of columella cells with laterally oriented PIN proteins.

Our data imply that basipetal transport and lateral redistribution of auxin are both critical for maintenance of the meristem zone. A potential mechanism to transport auxin to every cell in the meristem is indicated by PIN2 localization at basal membranes of cortex cells (Fig. 1e, f) and the expression domains of vascular PIN3 and PIN7, which suggest an 'auxin reflux' loop (Fig. 1h, j, n). We investigated auxin transport in the root by expressing the bacterial auxin biosynthetic enzyme IAAH under the WOX5 (ref. 24) promoter, which allows induction of auxin biosynthesis in the quiescent centre (Fig. 2G) by external addition of the precursor $\mathrm{IAM}^{25}$. After different time points of induction, we monitored auxin accumulation using the DR5-GFP reporter. In line with the observed polar orientation of the PIN proteins, enhanced auxin responses appear first in the columella region, subsequently in the lateral root cap and then in the epidermis; enhanced responses in provascular strands only emerge at later stages (Fig. $2 \mathrm{H}$, a-d). Epidermal and provascular auxin responses could be reduced by the polar transport inhibitor N-naphthylphthalamic acid (NPA), suggesting that accumulation of newly synthesized auxin in these regions is due to an NPA-sensitive auxin transport loop (Fig. $2 \mathrm{H}, \mathrm{e}$ ). In pin 2 mutant background the induced responses are restricted to the lateral root cap and epidermis, confirming a role for basipetal transport of auxin towards provascular cells in the root meristem (Supplementary Fig. 3).

Our results suggest that the capacity to circulate auxin through loop-oriented PIN efflux facilitators regulates meristem size. Such a loop system can redeploy auxin and hence operate at least partially independently from shoot auxin supply, consistent with the ability of isolated root systems to maintain growth without external auxin application. In a wider perspective, these data support that PIN protein localization predicts auxin transport routes.

PIN genes regulate cell expansion and root elongation zone size The capacity to stimulate cell expansion upon external application has been a defining property for auxins ${ }^{19}$. Recent analysis of tropic responses revealed that PIN genes play a part in differential auxin distribution, which is accompanied by differential cell expan$\operatorname{sion}^{7,9,11,26}$. It is, however, unknown whether PIN genes also regulate general cell expansion during organ growth. In roots, rapid cell expansion without cell division occurs in the elongation zone located proximal to the meristem zone (Fig. 2A).

Final cell size is affected in several pin mutants, but no additive effects occur in mutant combinations, suggesting that PIN gene action on cell expansion is complex. Nevertheless, in single allelic combinations of pin1pin3 and pin3pin7, the change in final cell length is the major factor accounting for the reduction in root length (Supplementary Fig 2, red fonts). In contrast to mature cell size, the size of cells immediately after departure from the meristem is not affected in any pin mutant combination (Supplementary Fig. 2).

We observed a reduction in the size of the elongation zone in pin mutant combinations, which mostly correlated well with reduction in meristem size. However, in pin3pin4pin 7 only the elongation zone size is reduced (Supplementary Fig. 2, blue fonts). These data 
indicate that the region where cell elongation occurs can be independently controlled by PIN gene activity.

Together, our data reveal that modulation of PIN activities can separately affect meristem size, elongation zone size and final cell size. These effects are not additive but probably result from interactions between changes in auxin distribution and transcriptional or translational responses influencing carrier components. We conclude that PIN-mediated modulation of auxin distribution controls both cell division and cell elongation and thereby contributes to the 'organizing' role of auxin in organ growth.

\section{PIN genes regulate PLT expression and pattern the distal root meristem}

Polar auxin transport is a major contributor to root meristem patterning in Arabidopsis, and the specification of distal cell types correlates well with the auxin response maximum ${ }^{1}$. Close to the auxin maximum, the quiescent centre maintains surrounding cells as stem cells (Fig. 1a). Quiescent centre and stem cell specification require SHR and SCR, putative GRAS family transcription factors $^{27,28}$ and the redundantly acting PLT1 and PLT2 AP2-domain putative transcription factors ${ }^{17}$. PLT transcript accumulation is
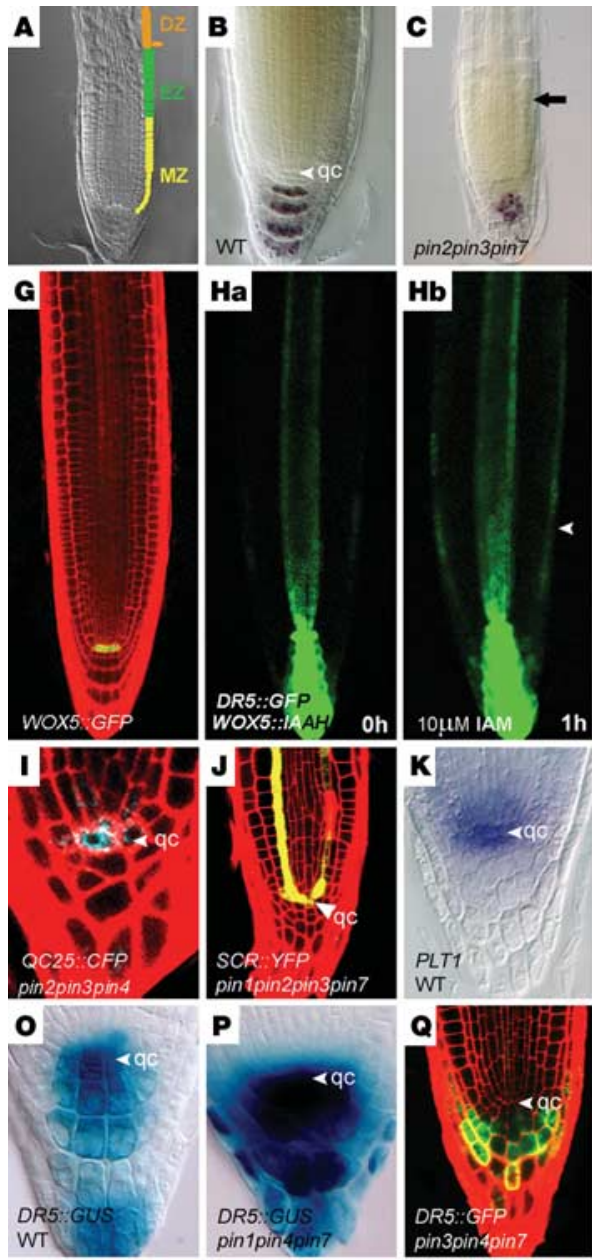

$\mathbf{u}$
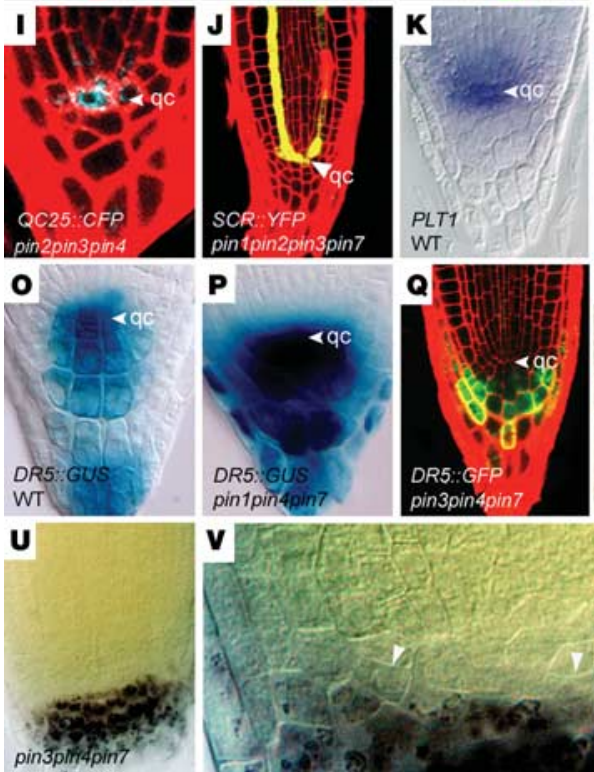

1
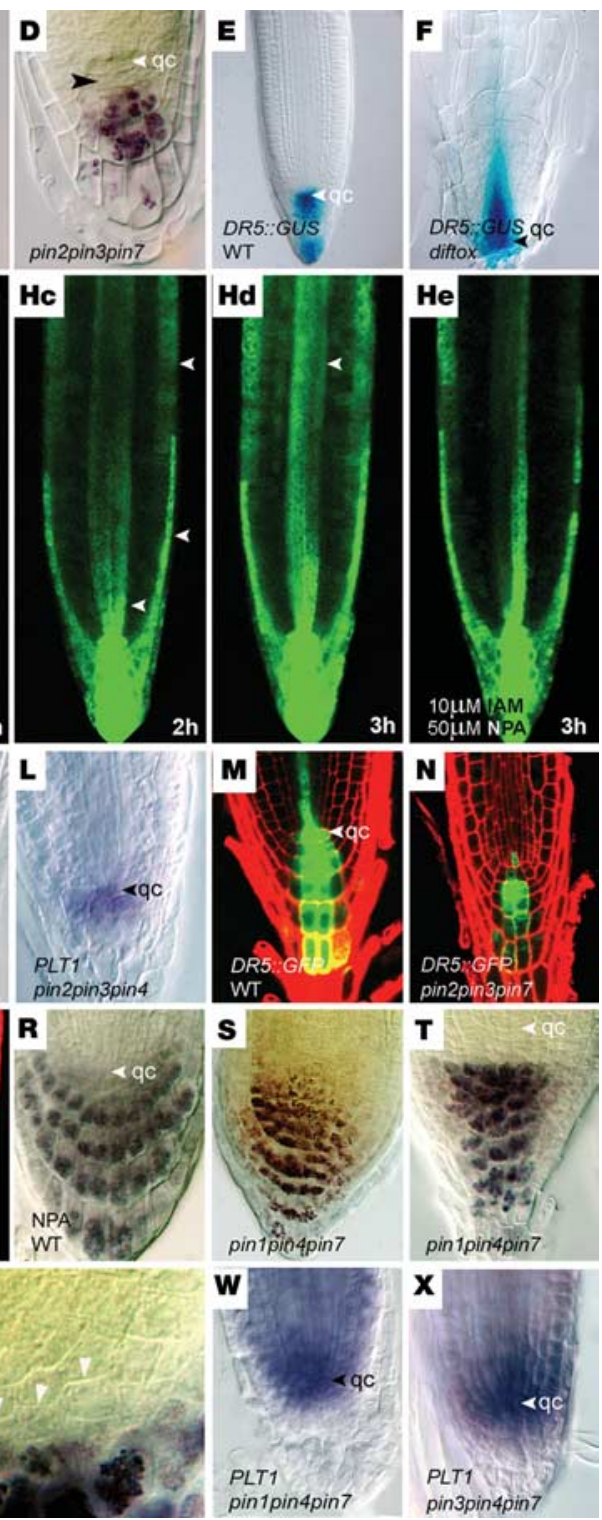

$\mathbf{T}$
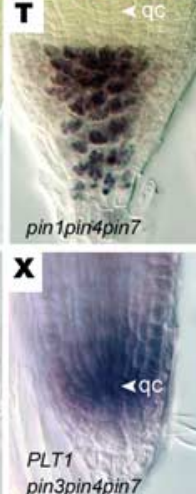

Figure 2 PIN genes control meristem size and patterning in Arabidopsis roots.

A-F, Meristem size control. A, Wild-type meristem zone (MZ), elongation zone (EZ) and

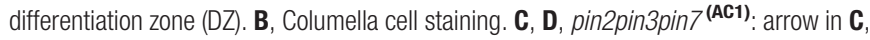
border of $\mathrm{MZ}$ and EZ; black arrowhead in $\mathbf{D}$, columella stem cells. E, F, DR5::GUS expression in wild type $(\mathbf{E})$ and $p R C P 1:: D T-A^{t s M}$ (root cap expressed diphtheria toxin, 'diftox') (F). G, WOX5 promoter specificity for quiescent centre. H, a-e, DR5::GFP in WOX5::IAAH plants. a, Control. b-d, DR5::GFP upregulation (arrowheads) after IAM application. e, No provascular upregulation on $50 \mu \mathrm{M} \mathrm{NPA}$. I-L, Patterning in pin2 mutant combinations. I, QC25::CFP (cyan fluorescent protein) in pin2pin3pin4 ${ }^{\text {(AC2) }}$. J, SCR::YFP (yellow fluorescent protein) in pin1pin2pin3pin ${ }^{{ }^{(A C 1)}} . \mathbf{K}, \mathbf{L}$, In wild type $(\mathbf{K})$ and in pin2pin3pin4 ${ }^{{ }^{(A C 2)}} \mathbf{( L )}, P L T 1$ transcript is restricted to the quiescent centre and stem cells.
$\mathbf{M}, \mathbf{N}, D R 5$ ::GFP in wild type (M) and pin2pin3pin ${ }^{(\mathbf{A C 1})}(\mathbf{N})$. $\mathbf{0}-\mathbf{X}$, pin mutants with patterning defects. Wild type treated with NPA (R). In $33 \%$ of pin1pin4pin $7^{\text {(AC1) }}$ DR5:: GUS (P), starch-granule-containing columella cells $(\mathbf{S})$ and $P L T 1$ expression (W) expand proximally (white arrowhead). In pin1pin4pin $7^{\text {(AC2) }}$, columella cells also expand laterally (T). In pin3pin4pin $7^{(\mathbf{A C 1})}$, mature columella cells (U), their stem cells (arrowheads in $\mathbf{V}$ ), DR5::GFP expression ( $\mathbf{Q})$ and PLT1 mRNA $(\mathbf{X})$ expand laterally. PLT1 transcript: wholemount in situ hybridization (blue/purple signals). Differentiated columella cells: starch granule staining (purple). DR5::GUS, blue. GFP fluorescence, green. qc, quiescent centre. $\mathbf{A}-\mathbf{F}, \mathbf{K}, \mathbf{L}, \mathbf{0}, \mathbf{P}, \mathbf{R}-\mathbf{X}$, Nomarski optics; $\mathbf{G}, \mathbf{H}, \mathbf{I}, \mathbf{J}, \mathbf{M}, \mathbf{N}, \mathbf{Q}, \mathrm{CLSM}$ after propidium iodide staining. 
correlated with auxin accumulation in embryos and primary and lateral roots, and depends on auxin response factors ${ }^{17}$. The identification of these critical factors for specification of distal cell types as well as the highly organized PIN gene expression domains in the

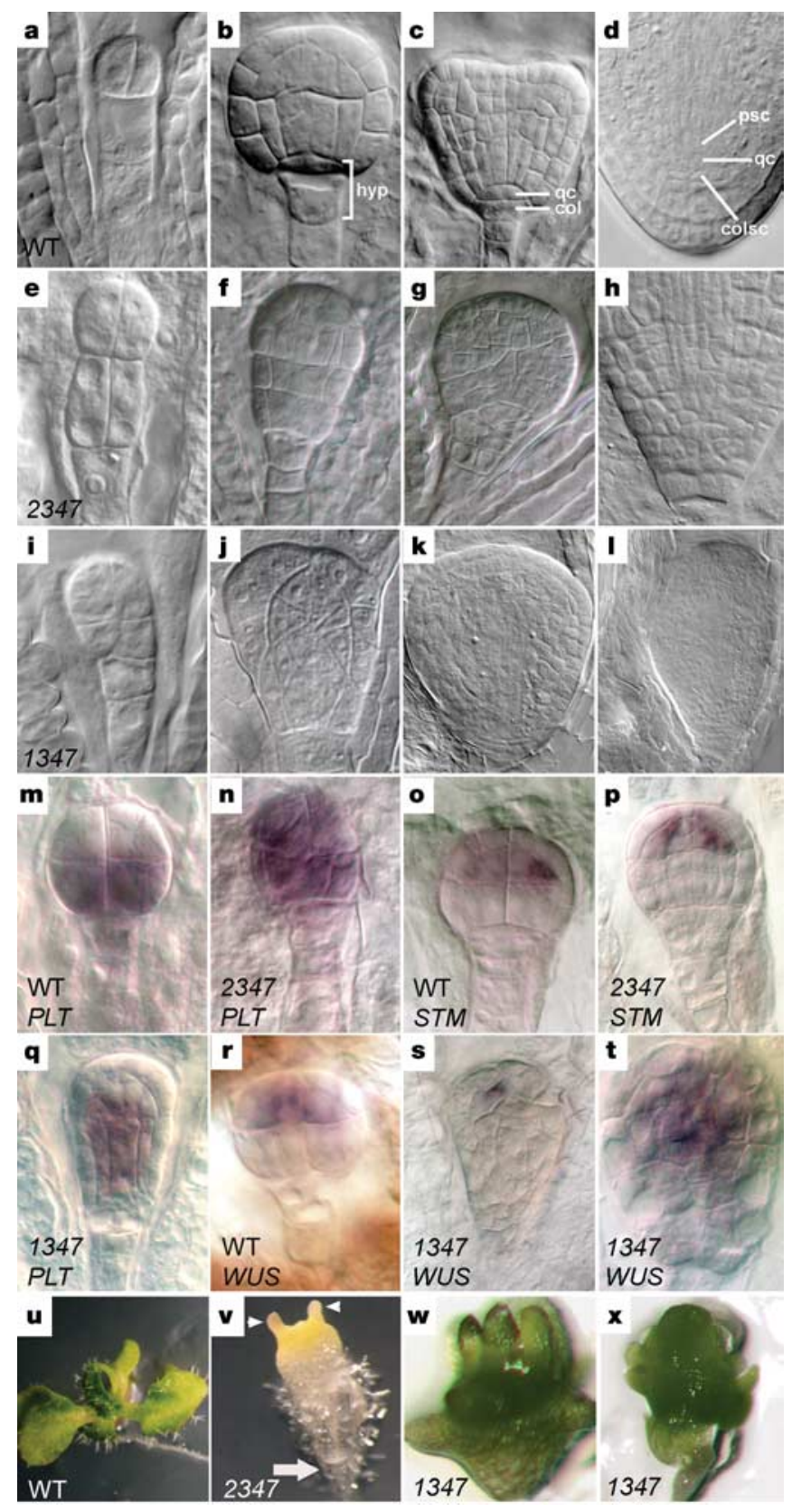

Figure 3 PIN genes and embryonic patterning. a-d, Wild-type embryo at 2-cell (a) globular (b) early heart (c) and torpedo stage (d). hyp, hypophysis; qc, quiescent centre; col, columella; sc, stem cells; psc, provascular stem cells. e-h, pin2pin3pin4pin7 ${ }^{\text {(AC1) }}$ embryos: e, aberrant divisions in basal cells at octant stage; $\mathbf{f}$, basal cell duplication at globular stage; $\mathbf{g}, \mathbf{h}$, quiescent centre and stem cell division defects at heart $(\mathbf{g})$ and torpedo stages (h). $\mathbf{i}-\mathbf{I}$, pin1pin3pin4pin $7^{\text {(AC1) }}$ embryos with abnormal basal (i) and apical cell divisions (j-l). $\mathbf{m}-\mathbf{t}$, Gene expression in pin quadruples: $\mathbf{m}, P L T 1$ mRNA in wild-type preglobular embryos is restricted to basal cells; $\mathbf{n}$, ubiquitous $P L T 1$ in

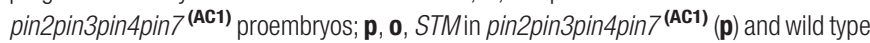
(o); $\mathbf{q}, P L T 1$ in pin1pin3pin4pin7 ${ }^{\text {(AC1) }} ; \mathbf{r}-\mathbf{t}$, In comparison to wild type (r), WUS is slightly reduced (s) or expands (t). $\mathbf{u}-\mathbf{x}$, Explanted embryos: $\mathbf{u}$, wild type develops normal seedlings; $\mathbf{v}$, pin2pin3pin4pin $7^{\text {(AC1) }}$ develops reduced cotyledons (arrowheads) and ectopic root hairs (arrow); w, $\mathbf{x}$, pin1pin3pin4pin7 ${ }^{\text {(AC1) }}$ explants have reduced root development and ectopic shoot-like structures at the apex (green tissues). PLT1, STM and WUS transcripts: in situ hybridization (purple). Images: Nomarski optics. root primordium set the stage for an analysis of the role of PIN genes in pattern formation.

In the previously discussed pin2 mutant combinations, meristem size is affected but distal patterning is normal, as judged by the presence of columella stem cells (Fig. 2D), the QC25 marker for the quiescent centre (Fig. 2I), SCR promoter activity (Fig. 2J), PLT1 (ref. 17) transcript distribution (Fig. 2K, L) and the DR5 auxin response marker (Fig. 2M, N).

In pin1pin4pin7, however, the auxin response maximum and the starch granules that mark the differentiated columella cells shift proximally in $40 \%$ of roots (Fig. 2P, S). In pin3pin 4 pin 7 , the auxin response maximum shifts laterally (Fig. 2Q), associated with inappropriate lateral up-regulation of PIN1 (Fig. 1c, d). Correlated with this lateral expansion, a broadening of the columella domain including its stem cells occurs (Fig. 2U, V). Consistent with changes in distal patterning, PLT1 messenger RNA shifts proximally in pin1pin4pin7 (Fig. 2W) and expands laterally in pin3pin4pin7 (Fig. 2X).

The phenotypes of both triple mutants resemble effects of treatment with inhibitors of polar auxin transport (Fig. 2R) and support a role for PIN proteins in focusing and stabilizing an auxin maximum in both proximo-distal and lateral dimensions. Our data strongly suggest that the PIN proteins in this way focus expression of the auxin-inducible PLT genes in the distal root region, which specifies the position of the quiescent centre and stem cells.

\section{PIN genes restrict PLT mRNA and root identity to the basal embryo pole}

We addressed whether PIN genes control PLT expression and root stem cell patterning during embryogenesis. Distal quiescent centre and columella cells originate from daughter cells of the hypophysis at the early globular stage of embryogenesis (Fig. 3a-d). At that stage an auxin perception maximum is detected in the hypophysis ${ }^{4}$.

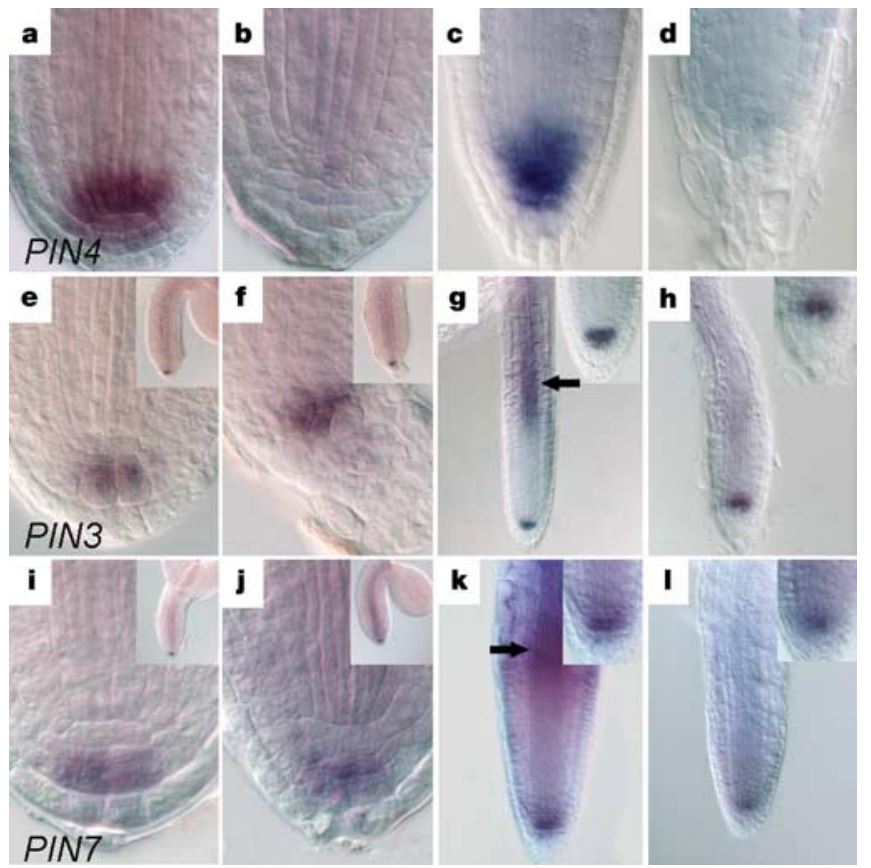

Figure 4 PLT genes regulate PIN transcript levels. a-d, PIN4 mRNA localization. PIN4 transcript in the quiescent centre, stem cells and provascular initials of wild-type embryo (a) and seedlings (c). No detectable transcripts in plt1plt2 double-mutant embryos (b) and seedlings (d). e-I, PIN3 and PIN7 mRNA in columella cells and provascular region of wildtype embryo (e, i) and seedlings (g, $\mathbf{k}$, arrows). plt1plt2 with normal levels in embryonic columella (f, j) and seedlings (h, I). Reduction of provascular expression in seedlings (h, I). Images: Nomarski optics. 
Clonal analysis and stereotyped cell division patterns show that the proximal stem cells are recruited around mid-heart stage (Fig. 3c $)^{29}$.

We found no penetrant embryonic defects in single, double and triple pin mutants and in pin1pin2pin3pin 7 and pin1pin2pin3pin4. However, in lines segregating pin2pin3pin4pin7 and pin1pin3pin4pin 7 mutants, marked changes were observed in embryonic division patterns and gene expression (Fig. 3e-t and Supplementary Table 2). pin2pin3pin4pin7 mutant embryos exhibit cell division defects mainly in the basal region (Fig. $3 \mathrm{e}-\mathrm{h}$ ) and $\sim 30 \%$ produce viable seedlings. In contrast, pin1pin3pin4pin 7 mutants are embryo lethal and cell division defects occur in apical and basal embryo regions ${ }^{4}$ (Fig. 3i-1). The significance of the altered cell divisions in both quadruple-mutant embryos in terms of patterning was investigated by locating the expression domain of marker genes for root and shoot identity. PLT1 transcript marks the basal domain of the octant stage embryo (Fig. $3 \mathrm{~m}$ ) and restricts to the quiescent centre and stem cell domain before mid-heart stage ${ }^{17}$. STM and WUS are required for shoot meristem function and are transcribed in shoot meristem precursor cells from early embryogenesis onwards ${ }^{30,31}$.

pin2pin3pin4pin7 mutants contain both aberrant cell divisions and high levels of PLT1 transcript throughout the embryo from the 16-cell stage onwards (Fig. 3n). In contrast, PLT1 mRNA is correctly excluded from the apical region in pin1pin3pin4pin7 mutants (Fig. 3q). Interestingly, the mRNA localization of WUS and STM does not change in pin2pin3pin4pin7 mutants (Fig. 3o, p), whereas in pin1pin3pin4pin7, WUS transcripts are either slightly reduced (Fig. 3s) or expanded in apical embryonic cells (Fig. 3t).

In explanted wild-type embryos, the shoot and root apical meristems develop normally (Fig. $3 \mathrm{u}$ ). In $\sim 40 \%$ of pin2pin 3 pin $4-$ pin7 mutants, the explants develop reduced cotyledons and root hairs emerge at more apical positions (Fig. 3v and Supplementary Table 2 ). In $\sim 80 \%$ of pin1pin3pin 4 pin 7 mutants, the explants completely arrest root growth and expand the shoot domain (Fig. 3w, $\mathrm{x}$ and Supplementary Table 2). The explant phenotypes of both mutants match the observed expansion of PLT1 and WUS domains, because ectopic expression of these genes promotes root and shoot identity, respectively ${ }^{17,32}$. PLT gene expression is strongly dependent on the joint action of PIN proteins, in line with its dependence on auxin response factors and the correlation between PLT expression and auxin accumulation ${ }^{17}$. Most probably the PLT expression domain is regulated by PIN4 and PIN7, which are

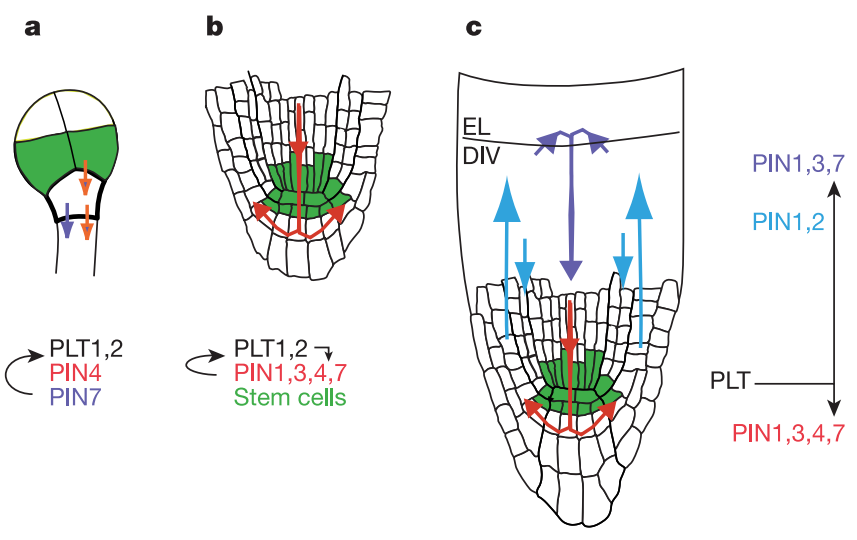

Figure 5 Model for primordium formation by PIN-PLT interactions. a, PIN-mediated root primordium specification by restriction of PLT transcripts in octant/16-cell embryo stage. b, At later stages of embryogenesis, PIN action further restricts PLT transcripts to define the stem cell region and PLT genes start controlling root-specific PIN gene expression. c, In post-embryonic roots, PIN-mediated auxin transport stabilizes the stem cell region and regulates cell division (DIV) in the meristem zone and cell expansion in the elongation zone (EL). PLT genes control several members of the PIN gene family to generate primordium-specific auxin distribution. appropriately positioned for basal auxin transport at the preglobular stage 4 . PIN3 and PIN2 are not expressed at this stage but ectopic PIN2 mRNA can be detected in pin3pin4pin7 siliques (data not shown), suggesting that ectopic expression of PIN members in embryos provides a remarkably versatile compensatory mechanism for the loss of PIN4 and PIN7.

PIN-regulated early PLT, WUS and STM transcription suggests that auxin transport in the embryo regulates the proper expression of critical root and shoot stem cell regulators. The notion that early cellular asymmetries in membrane localization of PIN proteins are translated into the patterning of embryonic stem cell domains via regulation of auxin flux provides a conceptual framework for initial events in plant embryogenesis.

\section{PLT genes regulate PIN gene expression in the root meristem}

The PLT genes are required for specification of the stem cell niche and convey root identity when ectopically expressed ${ }^{17}$. Therefore we asked whether the PLT proteins could regulate the root-specific distribution of PIN transcripts, thereby fine-tuning the position of the stem-cell-associated auxin maximum, and cell division and cell expansion domains. Remarkably, PIN4 transcript, which overlaps with the PLT transcripts in wild type, is undetectable in $94 \%$ and aberrant in $6 \%$ of the embryos and seedlings of plt1plt2 (Fig. $4 \mathrm{a}-\mathrm{d}$ ). PIN3 and PIN7 transcripts are normal in columella cells (Fig. 4e-1) but are markedly reduced in the provascular domain of the postembryonic root elongation zone (Fig. 4g, h, k, l). Thus, the PLT genes control PIN mRNA distribution.

\section{Discussion}

Our findings suggest an elegant mechanism for embryonic root primordium formation and stabilization. In our model, PIN proteins restrict PLT expression in the basal embryo region to initiate root primordium formation (Fig. 5a). In turn, PLT genes maintain PIN transcription, which stabilizes the position of the distal stem cell niche (Fig. 5b). At a distance from the auxin maximum, PLT genes maintain PIN3 and PIN7, which reinforce provascular acropetal auxin flux. In this way a 'reflux' loop is created that controls auxin distribution in the growing primordium and meristem (Fig. 5b, c). The loop stabilizes the auxin maximum and the PLTdependent stem cell domain in the distal root tip. Moreover, it localizes meristem and cell expansion zones in the proximal meristem and regulates final cell size (Fig. 5c).

Reporter genes and direct auxin measurements are consistent with the presence of a transport-regulated auxin gradient in the root meristem ${ }^{1}$, but local auxin biosynthesis and catabolism may also contribute to the auxin concentration profile ${ }^{33}$. Furthermore, auxin response may be regulated differently in the different root zones by processes such as differential SCF ${ }^{\mathrm{TIR} 1}$-mediated proteolysis ${ }^{34}$.

\section{Methods}

Materials

All pin mutants were in Columbia (Col-0) background, except for the Enkheim allele pin11. pin1-1, pin1En134; eir1-1, pin2En701; and pin3-3, pin4-2 were described in refs 7-10. pin3 salk_005544 and pin7 salk_048791 were provided by the Signal Insertion Mutant Library (http://signal.salk.edu/cgi-bin/tdnaexpress/). plt1-4 and plt2-2 alleles were described in ref. 17.

Triple mutants were generated by crossing double mutants sharing one allele, while quadruple mutants were generated by crossing triple homozygotes with two alleles in common. DR5::GUS (DR5- $\beta$-glucuronidase) was described in ref. 1 and was crossed with pin1, pin2, pin4 and pin7. Homozygous lines were then used to generate double and triple mutants homozygous for DR5::GUS. DR5-GFP, described in ref. 26, was transformed to wild type, pin2pin3pin7 and pin3pin4pin7. Promoter constructs of QC25::CFP and $S C R:: Y F P$ were transformed to pin2pin3pin4 and to pin1pin2pin3pin7 respectively. WOX5::GFP and WOX5::IAAH constructs were generated by fusing a $4.5-\mathrm{kb}$ WOX5 (ref. 24) promotor fragment in front of GFP or $I A A H^{25}$ in the pGreenII0229 (ref. 35) vector and transformed into the wild type or DR5::GFP lines.

\section{Phenotype analysis and microscopy}

Plant material for light microscopy was prepared as in ref. 36. Starch granules and $\beta$-glucoronidase activity were visualized as in ref. 36 . For embryo phenotype analysis, 
ovules were dissected and embryos were prepared as described in ref. 29. Embryo explant were cultured on petri dishes containing one-half GM medium and grown on the same medium for 4 weeks. For exogenous auxin application, wild type and multiple mutants were grown for 4 days (d) and transferred to medium supplemented with $100-200 \mathrm{nM}$ NAA for another $3 \mathrm{~d}$. Induction of auxin biosynthesis was performed by transferring plants to medium containing $10 \mu \mathrm{M}$ of the precursor IAM for $0-3 \mathrm{~h}$. Images were taken on a Zeiss Axioskop with a Nikon DXM1200 digital camera.

\section{In situ hybridization and immunolocalization}

For whole-mount in situ hybridization ${ }^{8}$, gene-specific 600-base-pair complementary DNA fragments for PIN4, PIN3 and PIN7 were used as probes. PLT1 probe was described in ref. 17 and STM and WUS probes were synthesized using the complete cDNA sequence. Immunolocalizations were performed on whole-mount roots of 5-7-day-old seedlings ${ }^{7,37}$. PINs antibodies and reagents were described in refs 5-8. Imaging was performed using a Leica SP2 inverted confocal microscope and accompanying software.

Received 5 July; accepted 5 November 2004; doi:10.1038/nature03184.

1. Sabatini, S. et al. An auxin-dependent distal organizer of pattern and polarity in the Arabidopsis root Cell 99, 463-472 (1999).

2. Benková, E. et al. Local, efflux-dependent auxin gradients as a common module for plant organ formation. Cell 115, 591-602 (2003).

3. Reinhardt, D. et al. Regulation of phyllotaxis by polar auxin transport. Nature 426, 255-260 (2003).

4. Friml, J. et al. Efflux-dependent auxin gradients establish the apical-basal axis of Arabidopsis. Nature 426, 147-153 (2003).

5. Gälweiler, L. et al. Regulation of polar auxin transport by AtPIN1 in Arabidopsis vascular tissue. Science 282, 2226-2230 (1998).

6. Müller, A. et al. AtPIN2 defines a locus of Arabidopsis for root gravitropism control. EMBO J. 17, 6903-6911 (1998)

7. Friml, J., Wisniewska, J., Benková, E., Mendgen, K. \& Palme, K. Lateral relocation of auxin efflux regulator PIN3 mediates tropism in Arabidopsis. Nature 415, 806-809 (2002).

8. Friml, J. et al. AtPIN4 mediates sink-driven auxin gradients and root patterning in Arabidopsis. Cell 108, 661-673 (2002).

9. Luschnig, C., Gaxiola, R. A., Grisafi, P. \& Fink, G. R. EIR1, a root-specific protein involved in auxin transport, is required for gravitropism in Arabidopsis thaliana. Genes Dev. 12, 2175-2187 (1998).

10. Utsuno, K., Shikanai, T., Yamada, Y. \& Hashimoto, T. Agr, an agravitropic locus of Arabidopsis thaliana, encodes a novel membrane-protein family member. Plant Cell Physiol. 39, 1111-1118 (1998).

11. Chen, R. et al. The Arabidopsis thaliana AGRAVITROPIC 1 gene encodes a component of the polar auxin-transport efflux carrier. Proc. Natl Acad. Sci. USA 95, 15112-15117 (1998).

12. Noh, B., Murphy, A. S. \& Spalding, E. P. Multidrug Resistance-like genes of Arabidopsis required for auxin transport and auxin-mediated development. Plant Cell 13, 2441-2454 (2001).

13. Noh, B., Bandyopadhyay, A., Peer, W. A., Spalding, E. P. \& Murphy, A. S. Enhanced gravi- and phototropism in plant $m d r$ mutants mislocalizing the auxin efflux protein PIN1. Nature 423, 999-1002 (2003).

14. Steinmann, T. et al. Coordinated polar localization of auxin efflux carrier PIN1 by GNOM ARF GEF. Science 286, 316-318 (1999)

15. Geldner, N., Friml, J., Stierhof, Y. D., Jürgens, G. \& Palme, K. Auxin transport inhibitors block PIN1 cycling and vesicle trafficking. Nature 413, 425-428 (2001).

16. Geldner, N. et al. Partial loss-of-function alleles reveal a role for GNOM in auxin transport-related, post-embryonic development of Arabidopsis. Development 131, 389-400 (2004).

17. Aida, M. et al. The PLETHORA genes mediate patterning of the Arabidopsis root stem cell niche. Cell 119, 109-120 (2004).

18. Sieberer, T. et al. Post-transcriptional control of the Arabidopsis auxin efflux carrier EIR1 require AXR1. Curr. Biol. 10, 1595-1598 (2000).
19. Srivastava, L. Plant Growth and Development: Hormones and Environment (Academic, New York, 2002)

20. Okada, K., Ueda, J., Komaki, M., Bell, C. \& Shimura, Y. Requirement of the auxin polar transport system in early stages of Arabidopsis floral bud formation. Plant Cell 3, 677-684 (1991).

21. Davies, P. J., Doro, J. A. \& Tarbox, A. W. The movement and physiological effect of indoleacetic acid following point applications to root tips of Zea mays. Physiol. Plant. 36, 333-337 (1976).

22. Rashotte, A. M., Brady, S. R., Reed, R. C., Ante, S. J. \& Muday, G. K. Basipetal auxin transport is required for gravitropism in roots of Arabidopsis. Plant Physiol. 122, 481-490 (2000).

23. Tsugeki, R. \& Fedoroff, N. V. Genetic ablation of root cap cells in Arabidopsis. Proc. Natl Acad. Sci. USA 96, 12941-12946 (1999).

24. Haecker, A. et al. Expression dynamics of WOX genes mark cell fate decisions during early embryonic patterning in Arabidopsis thaliana. Development 131, 657-668 (2004).

25. Kares, C., Prinsen, E., Van Onckelen, H. \& Otten, L. IAA synthesis and root induction with iaa genes under heat shock promoter control. Plant Mol. Biol. 15, 225-236 (1990).

26. Ottenschläger, I. et al. Gravity-regulated differential auxin transport from columella to lateral root cap cells. Proc. Natl Acad. Sci. USA 100, 2987-2991 (2003).

27. Di Laurenzio, L. et al. The SCARECROW gene regulates an asymmetric cell division that is essential for generating the radial organization of the Arabidopsis root. Cell 86, 423-433 (1996).

28. Helariutta, Y. et al. The SHORT-ROOT gene controls radial patterning of the Arabidopsis root through radial signaling. Cell 101, 555-567 (2000).

29. Scheres, B. et al. Embryonic origin of the Arabidopsis primary root and root meristem initials. Development 120, 2475-2487 (1994).

30. Mayer, K. F. et al. Role of WUSCHEL in regulating stem cell fate in the Arabidopsis shoot meristem. Cell 95, 805-815 (1998)

31. Long, J. A. \& Barton, M. K. The development of apical embryonic pattern in Arabidopsis. Development 125, 3027-3035 (1998).

32. Gallois, J. L., Nora, F. R., Mizukami, Y. \& Sablowski, R. WUSCHEL induces shoot stem cell activity and developmental plasticity in the root meristem. Genes Dev. 18, 375-380 (2004).

33. Ljung, K. et al. Biosynthesis, conjugation, catabolism and homeostasis of indole-3-acetic acid in Arabidopsis thaliana. Plant Mol. Biol. 50, 309-332 (2002).

34. Estelle, M. Auxin signaling and regulated protein degradation. Trends Plant Sci. 9, 302-308 (2004).

35. Hellens, R. P., Edwards, E. A., Leyland, N. R., Bean, S. \& Mullineaux, P. M. pGreen: a versatile and flexible binary Ti vector for Agrobacterium-mediated plant transformation. Plant Mol. Biol. 42 819-832 (2000).

36. Willemsen, V., Wolkenfelt, H., de Vrieze, G., Weisbeek, P. \& Scheres, B. The HOBBIT gene is required for formation of the root meristem in the Arabidopsis embryo. Development 125, 521-531 (1998).

37. Friml, J., Benkova, E., Mayer, U., Palme, K. \& Muster, G. Automated whole-mount localization techniques for plant seedlings. Plant J. 34, 115-124 (2003).

\section{Supplementary Information accompanies the paper on www.nature.com/nature}

Acknowledgements We thank N. Fedoroff and R. Tsugeki for seeds of $p R C P 1:: D T-A^{t s M}$; P. Hogeweg, S. Smeekens and members of the root laboratory for critical reading of the manuscript; and F. Kindt and R. Leito for artwork. K.P. acknowledges R. Nitschke and the Life Imaging Center (University of Freiburg). This work was supported by an NWO-PIONIER grant (I.B., J.X., M.W. and B.S.) and by the Deutsche Forschungsgemeinschaft and the FCI (I.P. and K.P.)

Competing interests statement The authors declare that they have no competing financial interests.

Correspondence and requests for materials should be addressed to B.S. (b.scheres@bio.uu.nl). 